\title{
Unusually High Prevalence of Stroke and Cerebral Vasculopathy in Hemoglobin SC Disease: A Retrospective Single Institution Study
}

\author{
Bindu Kanathezhath Sathi ${ }^{a}$ b Yilin Yoshidac Michael Raymond Weaver $^{b} d$ \\ Lila S. Nolan ${ }^{b, e}$ Barbara Gruner ${ }^{b}$ Vinod Balasa ${ }^{a}$ Talissa Altes ${ }^{b, f}$ \\ Carlos Leiva-Salinas ${ }^{f}$
}

aPediatric Hematology Oncology, Valley Children's Hospital, University of San Francisco-Fresno Program, Madera, CA, USA; ${ }^{b}$ Department of Child Health, University of Missouri, Columbia, SC, USA; 'Department of Internal Medicine, Tulane University, New Orleans, LA, USA; dUniversity of South Florida, Tampa, FL, USA; ${ }^{\mathrm{e} D e p a r t m e n t}$ of Pediatrics, Washington University, St. Louis, MO, USA; 'Department of Radiology, University of Missouri, Columbia, SC, USA

\author{
Keywords \\ Hemoglobin SC disease · Stroke · Silent cerebral infarction · \\ Cerebral vasculopathy
}

\begin{abstract}
Introduction: Unlike homozygous hemoglobin SS (HbSS) disease, stroke is a rare complication in hemoglobin SC (HbSC) disease. However, recent studies have demonstrated a high prevalence of silent stroke in $\mathrm{HbSC}$ disease. The factors associated with stroke and cerebral vasculopathy in the HbSC population are unknown. Methods: We conducted a retrospective study of all patients with sickle cell disease treated at the University of Missouri, Columbia, over an 18year period (2000-2018). The goal of the study was to characterize the silent, overt stroke, and cerebral vasculopathy in $\mathrm{HbSC}$ patients and compare them to patients with $\mathrm{HbSS}$ and $\mathrm{HbS} / \beta$ thalassemia1 (thal) in this cohort. We also analyzed the laboratory and clinical factors associated with stroke and cerebral vasculopathy in the HbSC population. Results: Of the $34 \mathrm{HbSC}$ individuals, we found that the overall prevalence of stroke and cerebral vasculopathy was $17.7 \%$. Only females had evidence of stroke or cerebral vasculopathy in our HbSC cohort $(33.3 \%, p=0.019)$. Time-averaged means of
\end{abstract}

karger@karger.com www.karger.com/aha

Karger"

BOPEN ACCESS
(C) 2021 The Author(s)

Published by S. Karger AG, Basel

This is an Open Access article licensed under the Creative Commons Attribution-NonCommercial-4.0 International License (CC BY-NC) (http://www.karger.com/Services/OpenAccessLicense), applicable to the online version of the article only. Usage and distribution for commercial purposes requires written permission. maximum velocities were lower in the $\mathrm{HbSC}$ group than the HbSS group and did not correlate with stroke outcome. Among HbSC individuals, those with stroke and cerebral vasculopathy had a marginally higher serum creatinine than those without these complications $(0.77 \mathrm{mg} / \mathrm{dL}$ vs. $0.88 \mathrm{mg} /$ $\mathrm{dL}, p=0.08)$. Stroke outcome was associated with recurrent vaso-occlusive pain crises (Rec VOCs) (75 vs. $25 \%, p=0.003$ ) in $\mathrm{HbSC}$ patients. The predominant cerebrovascular lesions in $\mathrm{HbSC}$ included microhemorrhages and leukoencephalopathy. Conclusion: There is a distinct subset of individuals with $\mathrm{HbSC}$ who developed overt, silent stroke, and cerebral vasculopathy. A female predominance and association with Rec VOCs were identified in our cohort; however, larger clinical trials are needed to identify and confirm specific clinical and laboratory markers associated with stroke and vasculopathy in HbSC disease.

(c) 2021 The Author(s) Published by S. Karger AG, Basell

\section{Introduction}

Children and adults with sickle cell disease (SCD) suffer from overt stroke, the prevalence of which has been reported to be $11 \%$ in individuals $<20$ years of age [1].
Correspondence to:

Bindu Kanathezhath Sathi, kanathezhathbindu@gmail.com 
More commonly, they suffer from silent cerebral infarction (SCI) that has a reported prevalence of $37.1 \%$ in children $<4$ years of age [2]. SCI is associated with neurocognitive deficits in children and is associated with subsequent risk of overt stroke [3-5].

Unlike homozygous sickle cell disease (HbSS), the reported prevalence of overt and silent stroke in hemoglobin SC (HbSC) disease is low, at 0 and 5.8\%, respectively, in Cooperative Study of Sickle Cell Disease (CSSD) [6]. However, in a recent study, the prevalence of SCI in HbSC was found to be $13.5 \%$ [7].

Given the association of SCI with both subsequent stroke [3] and overall decline in neurocognitive function [5], the detection and institution of comprehensive interventions, including judicious use of disease-modifying agents, are crucial [4]. Despite recent advances, both causal and progression factors associated with silent stroke and cerebral vasculopathy in $\mathrm{HbSS}$ disease are poorly understood. Comparatively lesser number of studies has been conducted looking into the prevalence, laboratory, and clinical factors associated with overt, silent stroke, and cerebral vasculopathy in HbSC disease, the second most common form of SCD. Currently, most of our understanding of HbSC is from extrapolation of data from HbSS disease [8].

The aim of our study was to characterize the silent and overt stroke, cerebral vasculopathy, and neurovascular complications in children and adults with $\mathrm{HbSC}$ and compare them to $\mathrm{HbSS}$ and $\mathrm{HbS} / \beta$ thalassemia (thal) in the Central Missouri Cohort (University of Missouri [MU]-SCD). Additionally, we examined the SCD-related complications and laboratory factors associated with cerebrovascular complications in this population.

\section{Methods}

In this retrospective cross-sectional study, pediatric and adult SCD patients treated at MU between 2000 (first availability of the electronic medical record) and 2018 were included. Retrospective chart review was conducted on all $\mathrm{HbSC}, \mathrm{HbSS}$, and $\mathrm{HbS} / \beta$ thal patients treated at MU medical center. The Institutional Review Board for MU, Columbia, MO, approved this study. Hemoglobin electrophoresis and, in some cases, $\beta$-globin gene sequencing was used to identify patients with $\mathrm{HbSS}, \mathrm{HbSC}$, and $\mathrm{HbS} / \beta$ thal. The classification of silent or overt stroke was done based on reported neurological events on chart review. Other SCD-related complications, such as pain crisis, acute chest syndrome, cutaneous ulceration, gallbladder disease, pulmonary hypertension, renal complications, and priapism, were reviewed throughout the course of the patient's medical history. SCD-related vaso-occlusive pain crises (VOC) were defined as an acute episode of pain that resulted in a visit to our medical facility and treatment with oral or parenteral narcotic medication or with a parenteral nonsteroidal anti-inflammatory drug.

Stroke and Vasculopathy in Hemoglobin SC
Recurrent VOC (Rec VOC) was defined as 2 or more episodes of VOCs warranting admission to the hospital for control of pain in 12 months preceding the identification of stroke or cerebral vasculopathy. Elevation in serum unconjugated (indirect) fraction of the bilirubin was defined as unconjugated hyperbilirubinemia. Indirect hyperbilirubinemia was defined as unconjugated bilirubin $>1.0 \mathrm{mg} / \mathrm{dL}$ at our institution.

The use of disease-modifying treatments with hydroxyurea (HU) and red blood cell exchange transfusion (RBCx) was assessed in all SCD individuals in the cohort. Children with SCD between ages 10-20 years with history of surveillance magnetic resonance imaging (MRI) scans were assessed for indications, including headache, seizures, and neurological deficits. All patients between the ages of 2 years-16 years also received annual transcranial doppler ultrasound (TCD) per institutional guidelines. TCD velocities were analyzed and verified by 2 independent radiologists on separate occasions. Three consecutive time-averaged means of maximum velocities (TAMMV) were obtained bilaterally at 3 locations in the middle cerebral artery (MCA) and 1 location in the anterior cerebral artery (ACA), as described previously [22]. Per institutional protocol, MRI was performed in SCD individuals if they had a history of headaches, neurological deficits, or an abnormal TCD. Clinical, laboratory, TCD, and MRI/ magnetic resonance angiography data for clinically documented overt stroke, SCI, and vasculopathy were analyzed.

Indication for MRI scanning for adults included transient or focal neurological events, severe headaches, or seizures. Routine surveillance MRI was not performed in adults at our institution. All MRIs were reviewed by a board-certified radiologist and rereviewed in a blinded manner by a neuroradiologist. The study neuroradiologist assessment was compared to the original report for agreement.

\section{Imaging Acquisition and Analysis}

Patients underwent MRI of the brain on Siemens Aera 1.5T or Skyra 3T MR scanners (Siemens Healthineers, Malvern, PA) with a standard 12-channel head coil. Sagittal three-dimensional magnetization prepared rapid acquisition gradient echo (MP-RAGE) T1-weighted, coronal T2-weighted, and axial fluid-attenuated inversion recovery (FLAIR), susceptibility-weighted, and diffusionweighted images were acquired as per the clinical protocols at the time of the examination. The neuroradiologist reviewed the MR images to classify the participants as having deep lacunar infarcts, encephalomalacia, brain atrophy, leukoencephalopathy/microvascular changes, parenchymal intracranial hemorrhage, chronic microhemorrhages, and superficial hemosiderosis.

We considered lacunes as small focal $<15 \mathrm{~mm}$ hyperintense/ CSF signal lesions in the basal ganglia or deep white matter of the brain. Such lacunes and encephalomalacia that were not associated with documented neurological deficits were termed as silent cerebral infarction [9]. Microhemorrhages were defined as punctate foci of hypo-intensity on susceptibility-weighted image and distinct from vascular flow voids and leptomeningeal hemosiderosis. The latter was considered when linear/curvilinear magnetic susceptibility within the subarachnoid space.

\section{Statistical Analysis}

Descriptive analysis was used and expressed as means +/- standard deviation (SD), medians (range), and numbers (\%). Fisher's exact tests for categorical data and Mann-Whitney U test for con- 
Table 1. Demographics of MU-SCD Cohort $(n, \%)$ by stroke status

\begin{tabular}{llll}
\hline & Stroke, $n(\%)$ & No stroke, $n(\%)$ & $p$ value \\
\hline Age, years & & & \\
$\leq 10$ & 0 & $14(100.0)$ & \\
$11-20$ & $10(32.3)$ & $21(67.7)$ & \\
$21-30$ & $7(25.9)$ & $20(74.1)$ & 0.025 \\
$31-40$ & $2(7.7)$ & $10(83.3)$ & \\
$41-50$ & $2(18.2)$ & $9(81.8)$ & \\
$\geq 51$ & $5(62.5)$ & $3(37.5)$ & \\
Gender & & & \\
Male & $13(24.1)$ & $41(75.9)$ & \\
Female & $13(26.5)$ & $36(73.5)$ & \\
Race/Country of origin & & & \\
African-American & $26(26.0)$ & $74(74.0)$ & \\
Jamaican & 0 & $2(100.0)$ & \\
Saudi Arabian & 0 & $1(100.0)$ & \\
Genotype & & & \\
HbSC & $6(17.7)$ & $28(82.4)$ & \\
HbSS & $18(30.0)$ & $42(70.0)$ & 0.475 \\
HbS/ $\beta$ thal & $2(22.2)$ & $7(77.8)$ & \\
\hline
\end{tabular}

HbSC, hemoglobin SC; HbSS, hemoglobin SS; SCD, sickle cell disease; MU, University of Missouri; thal, thalassemia.

tinuous data analysis were used. A $p$ value $<0.05$ was considered to be statistically significant. The analysis was conducted using PRISM and SAS software (9.3).

\section{Results}

Demographic Characteristics of the MU-SCD Cohort

We analyzed charts of 131 children and adults with SCD, with an age range of 1-65 years. On average, SCD patients received follow-up at MU for 1.7 decades of time at the medical center. Of the 131 individuals with SCD who sought care at MU, 28 had incomplete electronic medical data for assessment, and therefore, 103 patients were included in the statistical analysis.

We first determined the cerebrovascular changes in the HbSC group and compared them to the HbSS and $\mathrm{HbS} / \beta$ thal group. Of the 38 patients with $\mathrm{HbSC}, 34 \mathrm{pa}-$ tients had evaluable clinical data and 9 patients had prior brain MRI. In patients with HbSS disease, 60 patients had evaluable data and 30 patients had prior MRI. Of the 9 patients with $\mathrm{HbS} / \beta$ subtype, 5 patients had prior MRI of the brain.

No overt strokes were observed in children $<10$ years of age in the MU-SCD cohort (shown in Table 1). It was observed that $32 \%$ of children between 10 and 20 years
Table 2. Stroke and vasculopathy by SCD genotype ( $n, \%)$

\begin{tabular}{lllll}
\hline & HbSC & HbSS & $\begin{array}{l}\text { HbS/ } \\
\beta \text { thal }\end{array}$ & $\begin{array}{l}p \\
\text { value }\end{array}$ \\
\hline $\begin{array}{l}\text { Stroke and vasculopathy, n(\%) } \\
\quad \text { Yes }\end{array}$ & $6(17.7)$ & $18(30.0)$ & $2(22.2)$ & 0.476 \\
$\quad$ No & $28(82.3)$ & $42(70.0)$ & $7(77.8)$ & \\
$\begin{array}{l}\text { Stroke subcategory, n (\%) } \\
\quad \text { Overt stroke }\end{array}$ & $1(2.9)$ & $10(16.7)$ & $1(11.1)$ & \\
$\quad$ Silent stroke & $3(8.8)$ & $6(10.0)$ & $1(11.1)$ & \\
$\quad \begin{array}{l}\text { Silent stroke + vasculopathy } \\
\text { Vasculopathy }\end{array}$ & $2(5.9)$ & 0 & 0 & 0.473 \\
$\quad$ & 0 & $2(3.3)$ & & \\
$\quad$ No stroke & $28(82.4)$ & $42(70.0)$ & $7(77.8)$ & \\
\hline
\end{tabular}

$\mathrm{HbSC}$, hemoglobin SC; HbSS, hemoglobin SS; SCD, sickle cell disease; thal, thalassemia.

Table 3. Demographics of HbSC by stroke status in the MU-SCD cohort

\begin{tabular}{llll}
\hline & Stroke & No stroke & $p$ value \\
\hline $\begin{array}{l}\text { Gender, } n(\%) \\
\quad \text { Male }\end{array}$ & $0(0)$ & $16(100)$ & \\
$\quad \begin{array}{l}\text { Female } \\
\text { Age, } n(\%)\end{array}$ & $6(33.3)$ & $12(66.6)$ & 0.019 \\
$\quad$ Range (mean, y) & $11-66(36.17)$ & $3-60(20.43)$ & \\
$\quad<20 y$ & $3(13.6)$ & $19(86.3)$ & \\
$\quad>20 y$ & $3(25)$ & $9(75)$ & 0.19 \\
$\begin{array}{l}\text { Ethnicity, } n(\%) \\
\quad \text { African-American }\end{array}$ & $6(17.6)$ & $28(82.3)$ & \\
\hline
\end{tabular}

HbSC, hemoglobin SC; SCD, sickle cell disease; MU, University of Missouri.

had a cerebrovascular lesion, and the prevalence was highest compared to other age-groups. Among individuals with SCD surviving into the sixth decade of life, $62.5 \%$ had evidence of MRI lesions in the brain consistent with stroke. A significantly higher number of patients in the stroke category were on $\mathrm{HU}$ (76.9 vs. $23.1 \%, p=0.001)$ and $\mathrm{RBCx}$ therapy (61.5 vs. $38.5 \%, p=0.02)$.

\section{Stroke and Vasculopathy in HbSC Disease}

In the MU-SCD cohort, stroke and vasculopathy were observed in $17.7 \%(n=6)$ in the HbSC group. In comparison, stroke and vasculopathy were observed in $30 \%$ $(n=18)$ of the HbSS and $22.2 \%(n=2)$ of the $\mathrm{HbS} / \beta$ thal patients (shown in Table 2).

Silent stroke and cerebral vasculopathy were more frequently observed than overt stroke in the HbSC 
Table 4. Laboratory values among HbSC genotype patients by stroke status (mean, SD)

\begin{tabular}{|c|c|c|c|}
\hline Laboratory values (number) & $\begin{array}{l}\text { Stroke } \\
\text { mean (SD) }\end{array}$ & $\begin{array}{l}\text { No stroke } \\
\text { mean (SD) }\end{array}$ & $p$ value \\
\hline $\mathrm{Hgb}, \mathrm{g} / \mathrm{dL}$ (6 vs. 21$)$ & $10.41(0.51)$ & $10.85(1.16)$ & 0.38 \\
\hline Retic $\times 10^{9} /$ L (6 vs. 21$)$ & $4.19(1.89)$ & $3.76(1.52)$ & 0.56 \\
\hline $\mathrm{HbF}, \%$ (6 vs. 20$)$ & $1.36(1.65)$ & $12.58(5.01)$ & 0.23 \\
\hline WBC $\times 10^{9} /$ L (6 vs. 21$)$ & $11.75(4.11)$ & $10.36(2.95)$ & 0.35 \\
\hline Plt ( 6 vs. 21$) \times 10^{9} / \mathrm{L}$ & $249.3(21.41)$ & $284.3(98.13)$ & 0.44 \\
\hline Creatinine, mg/dL (6 vs. 21 ) & $0.77(0.23)$ & $0.58(0.22)$ & 0.08 \\
\hline $\mathrm{eGFR}, \mathrm{mL} / \mathrm{min} / 1.73 \mathrm{~m}^{2}$ (6 vs.21) & $108.1(26.5)$ & $122.3(17.3)$ & 0.13 \\
\hline Total bilirubin, mg/dL (6 vs. 20 ) & $1.21(0.91)$ & $1.46(0.74)$ & 0.48 \\
\hline AST, units/L (6 vs. 20$)$ & $42.63(26.30)$ & $34.32(16.63)$ & 0.35 \\
\hline
\end{tabular}

HbSC, hemoglobin SC; SD, standard deviation; AST, aspartate aminotransaminases; eGFR, estimated glomerular filtration rate.

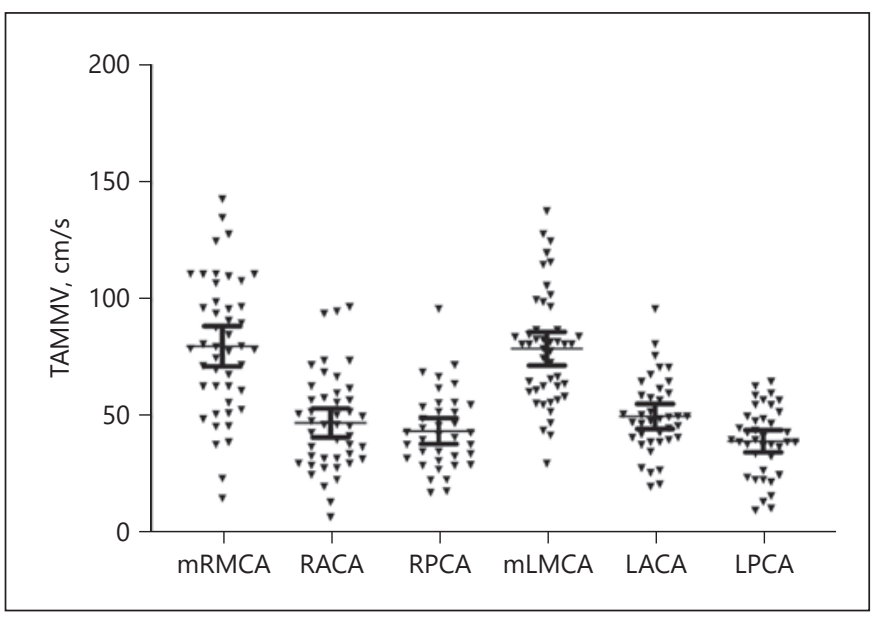

Fig. 1. TCD velocities in HbSC disease. TAMMV, timed averaged mean of maximum velocity; mRMCA, mid-right middle cerebral artery; RACA, right anterior cerebral artery, $\mathrm{rPCA}$, right posterior cerebral artery; mLMCA, mid-left middle cerebral artery; LACA, left anterior cerebral artery, LPCA, left posterior cerebral artery; HbSC, hemoglobin SC; TCD, transcranial Doppler ultrasound.

group (shown in Table 2). As expected, an overall higher prevalence of both silent and overt stroke was observed in the HbSS group than patients with HbSC and $\mathrm{HbS} / \beta$ thal. The percentage of cerebral vasculopathy was observed to be higher in HbSC than HbSS and $\mathrm{HbS} / \beta$ thal group in our cohort; however, these results were not statistically significant (shown in Table 2). Stroke and cerebral vasculopathy were identified only in women with HbSC in the MU-SCD cohort (33 vs. $0 \%, p=0.019$ ) (shown in Tables 3,5 ). All the patients with stroke or vasculopathy were of African-American ethnicity (shown in Table 3).

Stroke and Vasculopathy in Hemoglobin SC

\section{TCD Velocities and Stroke in MU-SCD}

As a part of MU institutional policy, TCD was routinely performed as part of annual surveillance in all children with SCD ( $\mathrm{HbSS}, \mathrm{HbSC}$, and $\mathrm{HbS} / \beta$ thal) $<16$ years of age (shown in online supplementary. Table 1; see www. karger.com/doi/10.1159/000519360 for all online suppl. material).

TCD was performed in $25(65.7 \%)$ patients with $\mathrm{HbSC}$ as a part of annual surveillance program in children. Three consecutive results were used to calculate the mean velocities as shown (shown in Fig. 1). The TAMMV for ACA, MCA, and posterior cerebral artery is shown (shown in Fig. 1). The TAMMV was $80.16 \mathrm{~cm} / \mathrm{s}[+/-$ $28.95 \mathrm{~cm} / \mathrm{s}$, SD and $4.46 \mathrm{~cm} / \mathrm{s}$, standard error of mean $(\mathrm{SEM})]$ in the right $\mathrm{MCA} ; 47.34 \mathrm{~cm} / \mathrm{s}(+/-20.61 \mathrm{~cm} / \mathrm{s}$, $3.072 \mathrm{~cm} / \mathrm{s}$; SD and SEM, respectively), in the right ACA and $43.86 \mathrm{~cm} / \mathrm{s}(+/-16.71 \mathrm{~cm} / \mathrm{s}$ and $2.74 \mathrm{~cm} / \mathrm{s}$; SD and SEM, respectively), in the right posterior cerebral artery. On the left side, the mean velocities were $79.12 \mathrm{~cm} / \mathrm{s}(+/-$ $24.17 \mathrm{~cm} / \mathrm{s}, 3.56 \mathrm{~cm} / \mathrm{s}$; SD and SEM, respectively) in MCA, $50.13 \mathrm{~cm} / \mathrm{s}(+/-16.4 \mathrm{~cm} / \mathrm{s}$ and $2.63 \mathrm{~cm} / \mathrm{s}$; SD and SEM, respectively) in the ACA and $39.53 \mathrm{~cm} / \mathrm{s}(+/-14.65$ $\mathrm{cm} / \mathrm{s}, 2.35 \mathrm{~cm} / \mathrm{s}$; SD and SEM, respectively).

No patient with HbSC had TAMMV above $170 \mathrm{~cm} / \mathrm{s}$ in this cohort (shown in Fig. 1). The left ACA was not visualized in 1 patient, but TAMMV was $<150 \mathrm{~cm} / \mathrm{s}$ in all other intracranial vessels. No elevation in TAMMV was observed in the preceding 12 months in 1 child with HbSC who developed overt stroke.

\section{Laboratory Parameters Associated with Stroke and Cerebral Vasculopathy in HbSC Disease}

The mean hemoglobin at steady state was above $10 \mathrm{~g} / \mathrm{dL}$ in both groups of patients with and without stroke in $\mathrm{HbSC}$ 

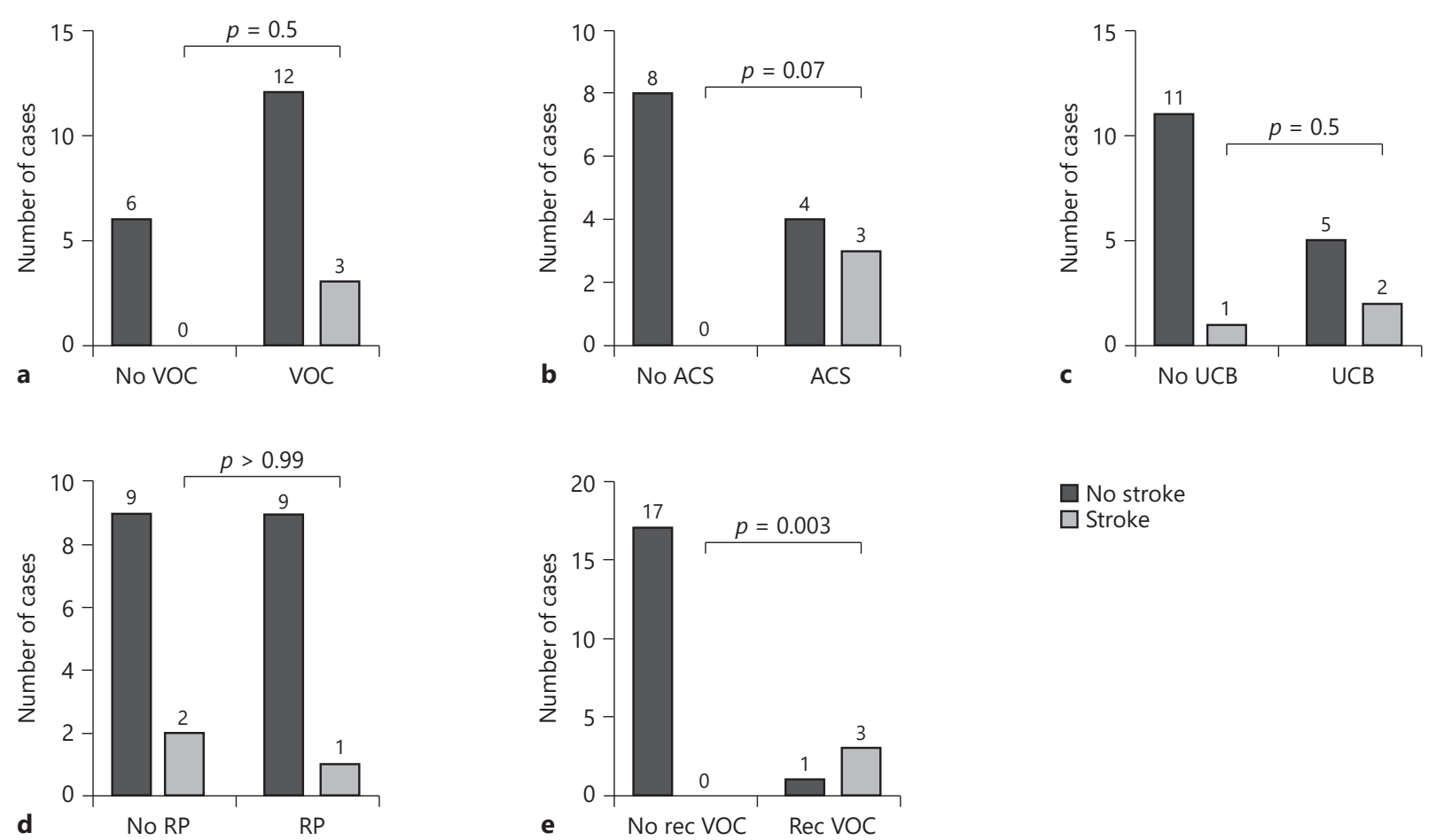

No stroke $\square$ Stroke

Fig. 2. Sickle cell-related complications associated with stroke. HbSC, hemoglobin SC; VOC, vaso-occlusive crisis; ACS, acute chest syndrome; UCB, unconjugated hyperbilirubinemia; RP, retinitis proliferans; Rec VOEs, recurrent vaso-occlusive episodes.

disease (shown in Table 4). Although not statistically significant, $\mathrm{HbF}$ was found to be lower in the group with stroke than those without a history of stroke. Higher serum creatinine was observed in individuals with stroke and vasculopathy than those who did not have stroke $(0.77 \mathrm{mg} / \mathrm{dL}$ vs. $0.88 \mathrm{mg} / \mathrm{dL}, p=0.08)$, but these findings were not statistically significant. In accordance with this finding, there was evidence of lower estimated glomerular filtration rate in the stroke and cerebral vasculopathy group than patients without stroke and cerebral vasculopathy in HbSC disease. Other laboratory parameters, including platelet count, white blood cell count, and aspartate aminotransaminases, were not statistically different in both groups.

\section{Sickle Cell-Related Complications Associated with \\ Stroke in HbSC Disease}

We then proceeded to analyze SCD-related complications associated with stroke outcome in the HbSC individuals (shown in Fig. 2). We observed that some laboratory markers of hemolysis (anemia, reticulocytosis, unconjugated hyperbilirubinemia, and increased urobilinogen) were not significantly different in the overt and silent stroke patients with HbSC compared to those without stroke (shown in Table 4). Complications associated with the hemolytic phenotype, such as pulmonary hypertension, priapism, cutaneous ulcer, and gallbladder stones were not statistically different in both groups. Osteonecrosis and proliferative retinopathy (62.5 vs. $37.5 \%, p=$ 0.9 ) of SCD were not associated with stroke outcome in HbSC disease (shown in Fig. 2d). Rec VOC in HbSC individuals was associated with stroke outcome compared to those without Rec VOC (75 vs. $25 \%, p=0.003$ ) (shown in Fig. 2e).

\section{MRI in HbSC Disease}

We analyzed the cerebrovascular lesions in patients in accordance with the criteria stated in the methods section. As brain MRI is not routinely performed in patients with $\mathrm{HbSC}$, clinical documentation was reviewed for determination of need for MRI. Of the 9 patients who obtained MRI, 8 had a history of severe, recurrent headaches, and 1 patient had a focal neurological deficit.

Among the HbSC patients with MRI, 1 (11.1\%) had lacunar infarcts (shown in Fig. 3a), 1 (11.1\%) showed fo- 


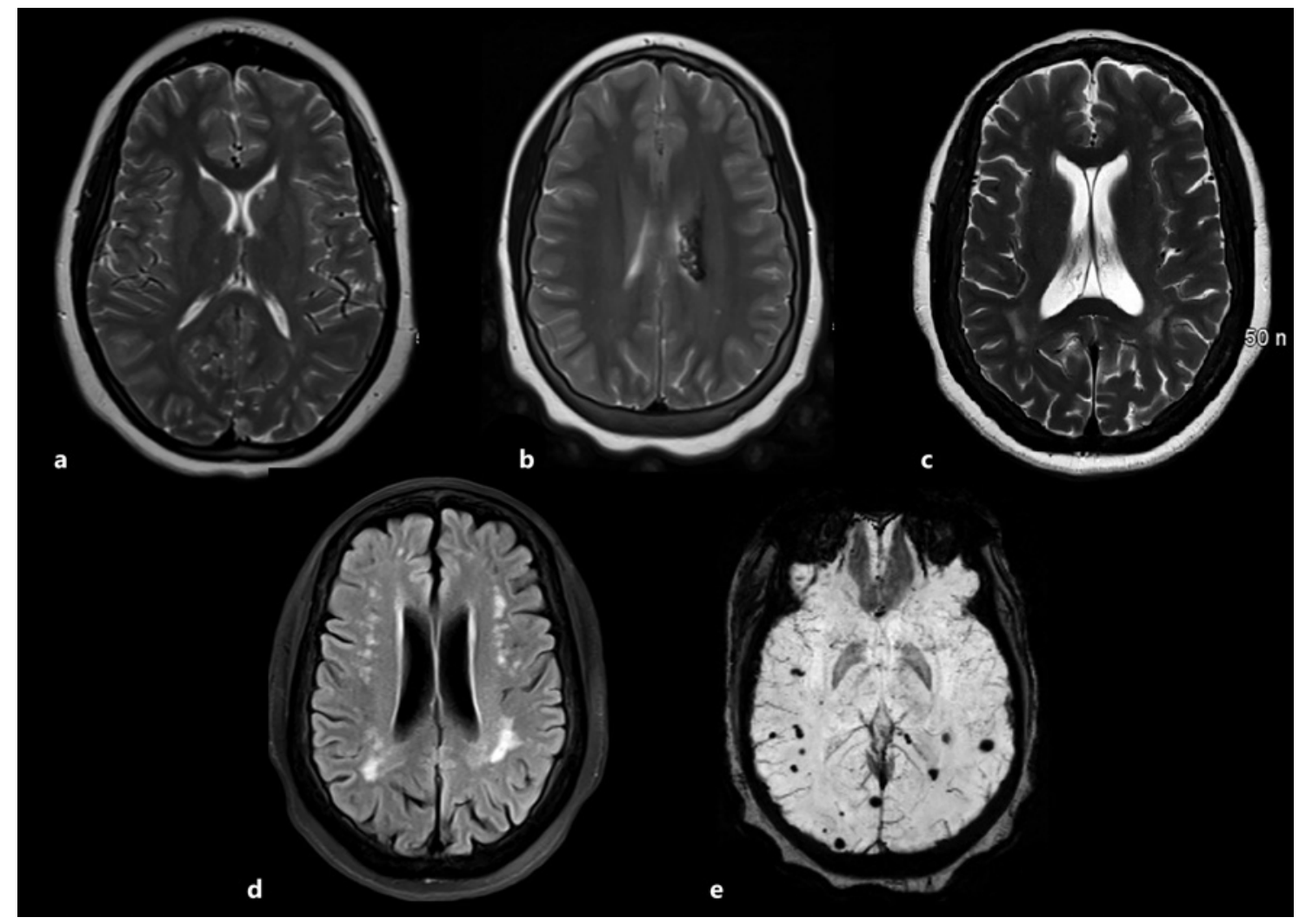

Fig. 3. Spectrum of MRI findings in patients with $\mathrm{HbSC}$ disease. MRI, magnetic resonance imaging; HbSC, hemoglobin SC; FLAIR, fluid-attenuated inversion recovery. a Axial T2-weighted image shows punctate hyperintense foci within the left caudate head and right medial thalamus, compatible with chronic lacunar infarcts. b Axial T2-weighted shows encephalomalacia and hemosiderin staining within the left periventricular white matter. c Axial T2weighted image shows global cerebral volume loss with mild ven-

cal encephalomalacia (shown in Fig. 3b), and 1 (11.1\%) cerebral atrophy (shown in Fig. 3c). The brain MRI revealed leukoencephalopathy in 3 (33.3\%) (shown in Fig. 3d) and microhemorrhages in 3 (33.3\%) (shown in Fig. 3e) subjects. No hemosiderosis was observed in HbSC patients in the cohort (online suppl. Table 2).

\section{Discussion}

The prevalence of SCI in HbSC has been reported to be around $5.8 \%$ compared to $21.8 \%$ in HbSS children between 6 and 18 years of age in the CSSD cohort $[1,6]$. More recent studies have shown that the prevalence of SCI in HbSC might be higher than previously reported [7]. As the number of individuals with HbSC obtaining routine brain MRI is low, it has been difficult to quantify triculomegaly. d Axial FLAIR image shows scattered punctate hyperintense foci within the white matter of the bilateral cerebral hemispheres, most compatible with chronic microvascular changes. e Axial susceptibility-weighted image shows multiple scattered foci of magnetic susceptibility within the subcortical white matter of the bilateral cerebral hemispheres consistent with chronic microhemorrhages.

the overall prevalence of silent stroke and cerebral vasculopathy in this population. Additionally, the clinical and laboratory variables associated with stroke in HbSC have not been previously studied. To address these gaps in our understanding, we retrospectively analyzed the clinical characteristics and laboratory and clinical parameters associated with both overt and silent stroke and vasculopathy in HbSC individuals.

Our study identified a higher than previously reported prevalence of SCI and cerebral vasculopathy in the HbSC population compared to previous CSSD reports [6]. SCI was more prevalent than overt stroke in the HbSC population, and interestingly similar white matter lesions have been reported in $1.3 \%$ of healthy children as incidental findings [10]. In this analysis, the overall prevalence of SCI and cerebral vasculopathy was around $14.7 \%$ whereas the prevalence of similar lesions was estimated to be 
Table 5. Characteristics of HbSC individuals with stroke and vasculopathy

\begin{tabular}{|c|c|c|c|c|c|c|c|}
\hline Patient & Age & Sex & Comorbidities & Stroke & Albuminuria & Treatment & Comments \\
\hline 1 & 13 & $\mathrm{~F}$ & VOC, rec VOC, and ACS & Overt stroke & No & $\begin{array}{l}\text { Exchange transfusion } \\
\text { Deferasirox }\end{array}$ & $\begin{array}{l}\text { Psychiatric symptoms, seizures, and poor } \\
\text { school performance }\end{array}$ \\
\hline 2 & 13 & $\mathrm{~F}$ & VOC and rec VOC & Silent stroke & No & $\mathrm{HU}$ & $\begin{array}{l}\text { Psychiatric symptoms, } \mathrm{HA} \text {, and poor } \\
\text { school performance }\end{array}$ \\
\hline 3 & 12 & $\mathrm{~F}$ & VOC and rec VOC & Silent stroke & No & $\mathrm{HU}$ & HA and poor school performance \\
\hline 5 & 65 & $\mathrm{~F}$ & ACS & Silent stroke/vasculopathy & Yes & $\begin{array}{l}\text { HU } \\
\text { ACE inhibitor }\end{array}$ & $\mathrm{HA}$ \\
\hline 6 & 64 & $\mathrm{~F}$ & Chronic renal failure & Silent stroke & Yes & $\begin{array}{l}\mathrm{HU} \\
\text { Dialysis }\end{array}$ & HA, seizures, died \\
\hline
\end{tabular}

HbSC, hemoglobin SC; VOCs, vaso-occlusive pain crises; Rec VOC, recurrent vaso-occlusive pain crisis; ACS, acute chest syndrome; HU, hydroxyurea.

$13.3 \%$ in the HbSS group which is in accordance with the prevalence that has been reported in other prospective studies [6]. The reported prevalence of cerebrovascular accidents by 20,30 , and 45 years of age was estimated to be 11,15 , and $24 \%$, respectively, in HbSS individuals but was significantly lower in the HbSC individuals at 2, 4, and $10 \%$, respectively [11]. Similar to our findings, some recent studies have also shown a higher incidence of SCI in $\mathrm{HbSC}$ population $[7,12,13]$. It is unclear if the wider use of high-resolution MRI is the cause of higher detection of these SCIs.

There were no dominant CNS lesions in HbSC disease compared to HbSS disease in our cohort. There was evidence of a slightly higher prevalence of encephalomalacia, atrophy, and microhemorrhage in HbSC than HbSS individuals (online suppl. Table 2). The most common MRI lesions reported in the brain of adults with HbSS disease has been leukoencephalopathy, followed by atrophy and encephalomalacia [14].

Our preliminary data indicate that the pathogenesis of stroke in patients with $\mathrm{HbSC}$ may be different from that seen in HbSS individuals. Patients with stroke or cerebral vasculopathy had mean hemoglobin above $10 \mathrm{~g} / \mathrm{dL}$, indicating that anemia is less likely a causative factor in these patients. Based on our observations, it is likely that hyperviscosity may play a role in the pathogenesis of both silent and overt strokes in HbSC individuals [15-18].

Although not statistically significant, higher concentration of $\mathrm{HbF}$ and lower creatinine were detected in the HbSC individuals without stroke than patients with stroke. Other indicators of hemolysis, including reticulocyte count, aspartate aminotransferase, and total biliru- bin, were not significantly different between the 2 groups. We did not routinely perform LDH testing on our patients with SCD, and therefore, these data were not available to us. Due to small size of study population, it is difficult to draw a definitive conclusion from these observations and more studies are needed.

In HbSS patients, both ACS and pain crises were previously observed to be negatively associated with silent stroke [19]. Anemia and hemolysis were found to be associated with silent stroke in HbSS, in previous studies [20]. In another study in HbSS individuals, it was found that SCI was associated with lower $\mathrm{Hb}$ and abnormal magnetic resonance angiography whereas VOC or acute chest syndrome did not positively correlate with stroke [20].

In our $\mathrm{HbSC}$ cohort, we found that patients with a previous history of Rec VOCs had a positive correlation with stroke outcome. However, the other sickle cell-related complications, like retinopathy, priapism, pulmonary hypertension, and cutaneous ulcer did not correlate with stroke outcome. Even though a single documented vasoocclusive episode did not correlate with stroke outcome, recurrent vaso-occlusive episodes were significantly associated with stroke and cerebral vasculopathy.

Factors known to play a role in HbSC disease pathology could in part play a role in HbSC stroke pathology. These include abnormal micro-and macrovascular function [21] and unique hemorheological properties of $\mathrm{HbSC}$ red blood cells (RBC) [22]. Impaired RBC deformability and increased RBC aggregation have been distinctly observed in HbSC disease [22].

TCD results did not correlate with stroke in HbSC, and the individual who developed overt stroke had nor- 
mal TCD in the preceding year. This lack of TCD correlation was a notable difference observed in HbSC patients compared to $\mathrm{HbSS}$ and $\mathrm{HbS} / \beta$ thal patients from historical trials $[23,24]$. Similar lack of correlation between TCD and SCI has been reported in other SCD cohorts $[20,25]$. On a broader perspective, TCDs have not been standardized in HbSC disease. TAMMV has been found to be significantly lower in HbSC $(<130 \mathrm{~cm} / \mathrm{s})$ than HbSS individuals from the Brazilian cohort [26, 27]. Further studies are warranted to assess if higher TAMMV is associated with overt or silent stroke in HbSC disease.

Stroke and vasculopathy were seen only in women in our HbSC cohort (shown in Table 5). This female predominance has not been noted in our HbSS cohort or in the historical HbSS controls [6]. Due to small numbers, it is difficult to ascertain at this time if female gender in itself contributes to the risk of stroke in HbSC disease.

Treatment considerations for overt and silent stroke in HbSC have mostly followed HbSS disease guidelines, even though the etiopathology of the disease might be different. Transfusion, HU, and phlebotomy have been undertaken in HbSC disease in small clinical trials [28-31]. Currently, there is an ongoing debate on the optimal management of SCI in SCD $[4,32]$. Furthermore, there are no available guidelines regarding the optimal management of SCI and overt stroke in HbSC individuals, and a multidisciplinary approach is warranted looking into all options, including stem cell transplantation, transfusions, phlebotomy, and HU [33].

Limitations of our study include the retrospective nature and the small number of patients who underwent MRI scans. There was also a selection bias as symptomatic patients were more likely to obtain brain MRI. Significant causal inferences would not be possible with small numbers and need to be tested in a larger prospective trial. Additionally, accurate prevalence cannot be ascertained by retrospective study and can be determined only by prospective screening. The incidence of stroke in our HbSC cohort was higher than what has been previously reported, but it is difficult to compare the incidence rate to historical controls by statistical analyses. There is also a possibility of ascertainment bias, with our radiologist using more advanced MRI techniques than historical cohorts, leading to higher detection rate of stroke and vasculopathy. Higher HU and RBCx use in the stroke category is likely to be confounded as an indication rather than cause-effect. More longitudinal studies are required to understand the onset and evolution of these cerebrovascular lesions in $\mathrm{HbSC}$ individuals.

Stroke and Vasculopathy in Hemoglobin SC
In conclusion, there is a recognizable subset of patients with HbSC who suffered from both silent and overt strokes. Stroke in HbSC has distinct differences compared to stroke in HbSS individuals. More studies are needed to understand the pathophysiology and risk factors associated with stroke in HbSC individuals. Earlier identification of at-risk individuals through clinical and laboratory biomarkers and institution of preventive and treatment strategies is warranted.

\section{Acknowledgments}

We would like to thank Ward Hagar, MD, for his editorial comments and Shalini Sathi, for editing the manuscript.

\section{Statement of Ethics}

This study was approved by the Institutional Review Board, University of Missouri- Columbia, MO, USA (IRB\#2004777 HS). Health Insurance Portability and Accountability Act (HIPAA) waiver of consent and assent was obtained for this study (MU IRB contact: cassanij@health.missouri.edu).

\section{Conflict of Interest Statement}

Speaker's Bureau (V.B.) for Novartis. Authors (B.K.S., Y.Y., M.R.W., L.N., B.G., T.A., C.L.S.) declare no competing conflict of interest.

\section{Funding Sources}

This project was funded by Missouri State Grant for Hemoglobinopathy (AOC17380120-B.K.S.) and Leda Sears Trust Fund, University of Missouri (B.K.S.). Y.Y. was partly supported by NIH grant (K12HDO43451) from Eunice Kennedy Shriver National Institute of Child and Human Development of Building Interdisciplinary Research Careers in Women's Health (BIRCWH) scholar. Some of the findings of this study were presented in abstract form at Annual American Society of Hematology Meeting, 2019.

\section{Author Contributions}

All authors contributed significantly to the article. B.K.S. and C.L.S. conceptualized the study, collected the data, and wrote the manuscript; B.G., V.B., and T.A. wrote and edited the manuscript; M.R.W. and L.N. collected the data and edited the manuscript; Y.Y. analyzed the data and edited the manuscript. 


\section{References}

1 Ohene-Frempong K. Stroke in sickle cell disease: demographic, clinical, and therapeutic considerations. Semin Hematol. 1991;28(3): 213-9.

2 Bernaudin F, Verlhac S, Arnaud C, Kamdem A, Chevret S, Hau I, et al. Impact of early transcranial Doppler screening and intensive therapy on cerebral vasculopathy outcome in a newborn sickle cell anemia cohort. Blood. 2011;117(4):1130-436; quiz 1436.

3 Miller ST, Macklin EA, Pegelow CH, Kinney TR, Sleeper LA, Bello JA, et al. Cooperative Study of Sickle Cell, silent infarction as a risk factor for overt stroke in children with sickle cell anemia: a report from the Cooperative Study of Sickle Cell Disease. J Pediatr. 2001; 139(3):385-90.

4 Quinn CT. Breakthrough: new guidance for silent cerebral ischemia and infarction in sickle cell disease. Hematology Am Soc Hematol Educ Program. 2014;2014(1):438-43.

5 White DA, Moinuddin A, McKinstry RC, Noetzel M, Armstrong M, DeBaun M. Cognitive screening for silent cerebral infarction in children with sickle cell disease. J Pediatr Hematol Oncol. 2006;28(3):166-9.

6 Pegelow CH, Macklin EA, Moser FG, Wang WC, Bello JA, Miller ST, et al. Longitudinal changes in brain magnetic resonance imaging findings in children with sickle cell disease. Blood. 2002;99(8):3014-8.

7 Guilliams KP, Fields ME, Hulbert ML. Higher-than-expected prevalence of silent cerebral infarcts in children with hemoglobin SC disease. Blood. 2015;125(2):416-7.

8 McCavit TL, Xuan L, Zhang S, Flores G, Quinn CT. National trends in incidence rates of hospitalization for stroke in children with sickle cell disease. Pediatr Blood Cancer. 2013;60(5):823-7.

9 Dowling MM, Quinn CT, Plumb P, Rogers ZR, Rollins NK, Koral K, et al. Acute silent cerebral ischemia and infarction during acute anemia in children with and without sickle cell disease. Blood. 2012;120(19):3891-7.

10 Kim BS, Illes J, Kaplan RT, Reiss A, Atlas SW. Incidental findings on pediatric MR images of the brain. AJNR Am J Neuroradiol. 2002; 23(10):1674-7.

11 Ohene-Frempong K, Weiner SJ, Sleeper LA, Miller ST, Embury S, Moohr JW, et al. Cerebrovascular accidents in sickle cell disease: rates and risk factors. Blood. 1998;91(1):28894.
12 Bindu K Sathi YY, Weaver MR, Nolan LW, Gruner B, Hirner C, Balasa VV, et al. Unusually high prevalence of silent stroke and cerebral vasculopathy in hemoglobin SC disease. Blood. 2019;134.

13 Houwing ME, Grohssteiner RL, Dremmen MHG, Atiq F, Bramer WM, de Pagter APJ, et al. Silent cerebral infarcts in patients with sickle cell disease: a systematic review and meta-analysis. BMC Med. 2020;18(1):393.

14 Silva GS, Vicari P, Figueiredo MS, Carrete H Jr, Idagawa MH, Massaro AR. Brain magnetic resonance imaging abnormalities in adult patients with sickle cell disease: correlation with transcranial Doppler findings. Stroke. 2009; 40(7):2408-12.

15 Johnson CS. Arterial blood pressure and hyperviscosity in sickle cell disease. Hematol Oncol Clin North Am. 2005;19(5):827-vi.

16 Pecker LH, Schaefer BA, Luchtman-Jones L. Knowledge insufficient: the management of haemoglobin SC disease. Br J Haematol. 2017; 176(4):515-26.

17 Lemonne N, Billaud M, Waltz X, Romana M, Hierso R, Etienne-Julan M, et al. Rheology of red blood cells in patients with $\mathrm{HbC}$ disease. Clin Hemorheol Microcirc. 2016;61(4):5717.

18 Lionnet F. Venous and arterial viscosity-associated thrombosis in hemoglobin SC disease. Am J Med. 2013;126(10):e13.

19 Kinney TR, Sleeper LA, Wang WC, Zimmerman RA, Pegelow CH, Ohene-Frempong K, et al. Silent cerebral infarcts in sickle cell anemia: a risk factor analysis. The Cooperative Study of Sickle Cell Disease. Pediatrics. 1999; 103(3):640-5.

20 Kwiatkowski JL, Zimmerman RA, Pollock AN, Seto W, Smith-Whitley K, Shults J, et al. Silent infarcts in young children with sickle cell disease. Br J Haematol. 2009;146(3):3005.

21 Mockesch B, Charlot K, Jumet S, Romana M, Divialle-Doumdo L, Hardy-Dessources MD, et al. Micro- and macrovascular function in children with sickle cell anaemia and sickle cell haemoglobin C disease. Blood Cells Mol Dis. 2017;64:23-9.

22 Nagel RL, Fabry ME, Steinberg MH. The paradox of hemoglobin SC disease. Blood Rev. 2003; 17(3):167-78.

23 Ware RE, Davis BR, Schultz WH, Brown RC, Aygun B, Sarnaik S, et al. Hydroxycarbamide versus chronic transfusion for maintenance of transcranial doppler flow velocities in children with sickle cell anaemia-TCD with transfusions changing to hydroxyurea (TWiTCH): a multicentre, open-label, phase 3, non-inferiority trial. Lancet. 2016; 387(10019):661-70.
24 Adams RJ, Brambilla DJ, Granger S, Gallagher D, Vichinsky E, Abboud MR, et al. Stroke and conversion to high risk in children screened with transcranial Doppler ultrasound during the STOP study. Blood. 2004; 103(10):3689-94.

25 Wang WC, Gallagher DM, Pegelow $\mathrm{CH}$, Wright EC, Vichinsky EP, Abboud MR, et al. Multicenter comparison of magnetic resonance imaging and transcranial Doppler ultrasonography in the evaluation of the central nervous system in children with sickle cell disease. J Pediatr Hematol Oncol. 2000;22(4): 335-9.

26 Vieira C, de Oliveira CN, de Figueiredo LA, Santiago RP, Adanho CS, Santana SS, et al. Transcranial Doppler in hemoglobin SC disease. Pediatr Blood Cancer. 2017;64(5).

27 Santiago RP, Vieira C, Adanho CSA, Santana SS, Guarda CC, Figueiredo CVB, et al. Laboratory and genetic biomarkers associated with cerebral blood flow velocity in hemoglobin SC disease. Dis Markers. 2017;2017:6359871.

28 Fridlyand D, Wilder C, Clay ELJ, Gilbert B, Pace BS. Stroke in a child with hemoglobin SC disease: a case report describing use of hydroxyurea after transfusion therapy. Pediatr Rep. 2017;9(1):6984.

29 Luchtman-Jones L, Pressel S, Hilliard L, Brown RC, Smith MG, Thompson AA, et al. Effects of hydroxyurea treatment for patients with hemoglobin SC disease. Am J Hematol. 2016;91(2):238-42.

30 Miller MK, Zimmerman SA, Schultz WH, Ware RE. Hydroxyurea therapy for pediatric patients with hemoglobin SC disease. J Pediatr Hematol Oncol. 2001;23(5):306-8.

31 Markham MJ, Lottenberg R, Zumberg M. Role of phlebotomy in the management of hemoglobin SC disease: case report and review of the literature. Am J Hematol. 2003;73(2): 121-5.

32 King A, Herron S, McKinstry R, Bacak S, Armstrong M, White D, et al. A multidisciplinary health care team's efforts to improve educational attainment in children with sickle-cell anemia and cerebral infarcts. J Sch Health. 2006;76(1):33-7.

33 DeBaun MR, Armstrong FD, McKinstry RC, Ware RE, Vichinsky E, Kirkham FJ. Silent cerebral infarcts: a review on a prevalent and progressive cause of neurologic injury in sickle cell anemia. Blood. 2012;119(20):4587-96. 